Ann. Biol. anim. Bioch. Biophys., I970, 10 (3), 48 1-492.

\title{
ÉVOLUTION DE LA TENEUR EN ACIDES NUCLÉIQUES DE LA MUSCULATURE ÉPAXIALE AU COURS DE LA CROISSANCE CHEZ LA TRUITE ARC-EN-CIEI, (SALMO GAIRDNERII); RÔLES RESPECTIFS DE LA MULTIPLICATION ET DU GRANDISSEMENT CELLULAIRES
}

\author{
P. LUQUET et G. DURAND \\ avec la collaboration technique de Geneviève Hannequart \\ Station centrale de Nutrition. \\ Centre national de Recherches zootechniques, 78 -Jouy-en-Josas \\ Institut national de la Recherche agronomique
}

\section{RÉSUMÉ}

La croissance de la musculature épaxiale de la truite Arc-en-ciel a été étudiée en utilisant les tests suivants:

- mesure des variations de la teneur en acide désoxyribonucléique (ADN), dont la quantité est proportionnelle au nombre de cellules:

- mesure des variations du rapport : poids frais/ADN, proportionnel à la taille des cellules;

- mesure des variations du rapport : acide ribonucléique/ADN qui donne un aspect de l'activité cellulaire.

Lorsque le poids des truites est multiplié par 163 (croissance de $20 \mathrm{~g}$ à $3400 \mathrm{~g}$ ) il est montré que :

- le poids du muscle est multiplié par ${ }^{6} 65$,

- le nombre de cellules est multiplié par 78 ; la multiplication cellulaire (hyperplasie) est continue tout au long de l'intervalle étudié,

- les cellules ont doublé de taille; elles ont atteint 75 p. roo de leur taille finale dès que les truites pèsent $400 \mathrm{~g}$, poids à partir duquel le grandissement cellulaire (hypertrophie) se ralentit,

- l'hyperplasie participe toujours pour plus de 7o p. Ioo à l'accroissement pondéral du muscle. Son intervention relative est supérieure à $90 \mathrm{p}$. Ioo lorsque le poids des truites dépasse $400 \mathrm{~g}$.

Ces résultats sont comparés à ceux obtenus chez le Rat et chez le Porc. Ils indiquent que les mécanismes de la croissance des poissons diffèrent sensiblement de ceux qui régissent la croissance de ces mammifères. 


\section{INTRODUCTION}

Les lois qui régissent la croissance des poissons sont encore mal établies. Divers auteurs se sont basés sur les modifications de longueur et de poids en fonction de l'âge, et sur les niveaux métaboliques correspondants mesurés par la consommation d'oxygène, mais arrivent cependant à des conclusions sensiblement divergentes. En effet, si VON BERTALANFFy (Ig60) propose une forme de croissance semblable à celle connue chez les mammifères, les courbes établies par PALOHEIMo et DIAKIE (I965) suggèrent que "le poids du corps continue à croître tout au long de la vie des poissons et atteindrait théoriquement l'infini dans un temps infini ". Par ailleurs, il n'existe pratiquement pas de données sur les mécanismes qui régissent la croissance des poissons ; c'est pour cette raison que nous avons entrepris un travail destiné à étudier le type de déroulement de la croissance de la Truite au moyen de tests biochimiques.

Pour ce faire, nous avons adopté tune méthode basée sur les principes établis par BoIvin et VENDRELEY (I948) et utilisée d'abord par ENESCO (I959) puis reprise en particulier par Strunz et LenkeIt (I963), Moss et al. (I964), DUR.AND et al. (I965). Cette méthode permet de mesurer l'évolution du nombre de cellules par l'évolution de la quantité totale d'acide désoxyribonucléique (ADN) dans un tissu ou un organe. La taille des cellules, définies comme l'ensemble d'un noyau et de la quantité de cytoplasme qu'il contrôle, se mesure par le rapport du poids frais du tissu ou de l'organe à sa teneur totale en ADN.

\section{MATÉRIEL E'T MÉTHODES}

\section{I. - Matériel animal}

a) Composition des lots.

Huit lots de truites Arc-en-Ciel (Salmo gairdneri) de même souche ct élerées en pisciculture ont été constitués. Jusqu'au poids de $50 \mathrm{~g}$ les poissons ont consommé un aliment commercial contenant $49 \mathrm{p}$. Ioo de protéines à raison de $3,5 \mathrm{p}$. Ioo du poids vif par jour. Au-delà du poids de $50 \mathrm{~g}$, les truites recevaient un aliment contenant $45 \mathrm{p}$. 100 de protéines à raison de 2,5 à $\mathrm{I}$ p. I0O de leur poids vif par jour. La température d'élevage variait suivant les saisons entre $8^{\circ} \mathrm{C}$ et $2 \mathrm{I}^{\circ} \mathrm{C}$. Le nombre d'animaux constituant chaque lot et leur poids moyen correspondant figurent dans le tableau $\mathrm{I}$.

TABI, EAU I

Caractéristiques des lots

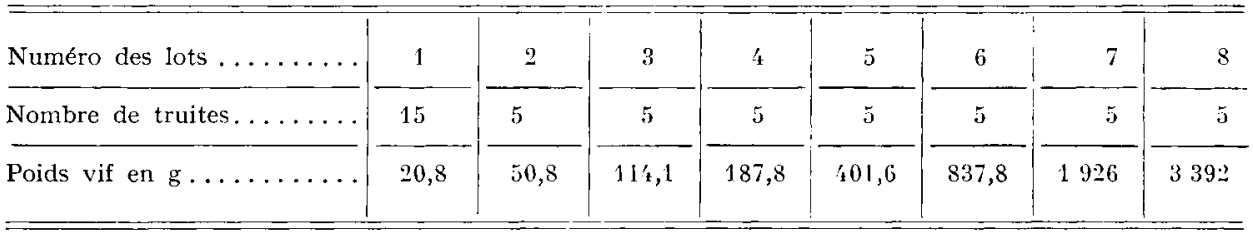

Les lots de I à 5 comportent des poissons en croissance sacrifiés au cours des mois d'été. Les lots 6 , 7 et 8 sont constitués de truites mâles abattues au cours des mois d'hiver ; l'examen des gonades indique que ces animaux sont en état de maturité sexuelle. 
La musculature de chaque poisson a été traitée individuellement pour les analyses, excepté les muscles des truites du lot I qui ont été regroupées par 3 car les techniques d'analyse nécessitent au moins $15 \mathrm{~g}$ de tissus frais.

\section{b) Sacrifice des truites et prélèvement du muscle.}

Chaque truite est anesthésiée par immersion dans un bain de MS 222 Sandoz (tricaine métasulfonate) puis séchéc au papier-filtre et pesée. Elle est ensuite saignée par incision de l'aorte ventrale : $3 \mathrm{ml}$ de sang par Ioo $\mathrm{g}$ de poids vif sont recueillis en moyenne. La peau est enlevée, puis les deux filets latéraux dorsaux (musculature épaxiale) sont prélevés en totalité aussi rapidement que possible ; l'ensemble des opérations décrites ci-dessus s'effectue en zo minutes environ. Après pesée, les filets sont congelés immédiatement par immersion dans de l'azote liquide et conservés dans ce fluide jusqu'aux opérations ultérieures ( 36 heures au maximum.) Les muscles dont le poids excède $100 \mathrm{~g}$ sont broyés finement à froid et une quantité de $70 \mathrm{~g}$ est retenue pour les analyses.

\section{II. - Méthodes analytiques}

Les méthodes analytiques utilisées sont celles décrites par I)URAND et al. (I965, I969). Les principales étapes en sont: $\left(\mathrm{O}^{\circ}-2 \mathrm{OC}\right)$

- l'extraction des composés acido-solubles par l'acide trichloracétique à Io p. Ioo à froid

- l'extraction des lipides par le mélange méthanol-chloroforme ( $\mathrm{r}$ vol/2 vol.);

- le lavage du résidu à l'alcool $100^{\circ}$, puis à l'éther sulfurique ;

... le séchage du résidu sous vide;

- la séparation des acides nucléiques par la méthode de Schmid et Thannhauser (I9+5);

- la séparation chromatographique des monoribonucléotides d'une part et des bases puriques de l'ADN d'autre part sur des colonnes échangeuses d'ions ;

- l'estimation de ces composés par spectrophotométrie dans l'UV.

\section{RÉSULTATS}

\section{I. - Mode d'expression}

Les résultats sont rapportés au poids frais du muscle. Le poids frais, estimé par pesée après dissection, diffère légèrement du poids réel. En effet, au cours du prélèvement, il se produit une évaporation d'une petite quantité d'eau; par ailleurs, le muscle contient un peu de sang résiduel. Cependant, comme le mode de prélèvement est toujours identique, cette méthode permet de mesurer l'évolution de la croissance pondérale du muscle d'une façon satisfaisante. Le poids sec et dégraissé (PSD) est obtenu directement pour les muscles des truites des cinq premiers lots. Pour les lots 6,7 et 8 , le PSD est estimé d'après le pourcentage par rapport au poids trais calculé par pesée avant et après la série d'extractions effectuées sur le prélèvement de $70 \mathrm{~g}$. Le PSD contient essentiellement des protéines (90 p. I00), les acides nucléiques et une petite quantité de minéraux. L'évaluation du rapport PSD/poids frais permet donc d'apprécier assez exactement la teneur en protéines du muscle. Les quantités d'ARN sont exprimées par la somme des monoribonucléotides (en micromoles) constitutifs ; la proportion de ces différents nucléotides a déjà été donnée par ailleurs (LUQUET I970). Les quantités d'ADN sont exprimées par la somme des bases puriques (en micromoles) multipliée par 2. En effet, d'après CharGaFF et LIPSHITZ (I953) la somme des bases puriques est égale à la somme des bases pyrimidiques. Cette loi a d'ailleurs été vérifiée récemment par BERDYSHEV et al. (I967) dans divers tissus et organes du Saumon. La conversion des résultats en $\gamma$ de P-ARN et $\mathrm{P}-\mathrm{ADN}$ peut être effectuée simplement en multipliant les résultats ainsi exprimés par $3 \mathrm{I}$. 


\section{II. - Évolution du poids frais et du poids sec et dégraissé du muscle}

Lorsque le poids vif des truites augmente de $20 \mathrm{~g}$ à $3400 \mathrm{~g}$, il y a en moyenne isométrie de croissance entre le muscle et l'organisme en entier. Le poids de la musculature épaxiale est en effet multiplié par I 65 alors que celui de la truite l'est par I63. Toutefois, tel que cela ressort de la figure I, cette isométrie n'est pas rigoureusement observée pour les différents intervalles successifs. En effet, si le coefficient d'allométrie est pratiquement égal à I dans les intervalles de poids $20 \mathrm{~g}-50 \mathrm{~g}$ et $\mathrm{I} 90 \mathrm{~g}-400 \mathrm{~g}$, il n'est égal qu'à 0,80 dans l'intervalle $400 \mathrm{~g}$ - $840 \mathrm{~g}$ tandis qu'il a pour valeur $\mathrm{I}, 3 \mathrm{I}$ dans l'intervalle Ir $5 \mathrm{~g}$ - I $90 \mathrm{~g}$. Le poids sec et dégraissé du muscle augmente en général plus vite que le poids frais de ce tissu sauf entre le poids de $400 \mathrm{~g}$ et I $93^{\circ} \mathrm{g}$. Au cours de cet intervalle en effet le poids frais est multiplié par 4,I alors que le poids sec et dégraissé n'est multiplié que par 3,3.

\section{III. - Évolution de la teneur du muscle en acides nucléiques}

a) Évolution de la concentration en $A R N$ et en $A D N$ du muscle sec et dégraissé (fig. 2, $\mathrm{A}$ et $\mathrm{B}$ )

La quantité d'ADN contenue par gramme sec et dégraissé diminue de moitié lorsque le poids des truites augmente de $20 \mathrm{~g}$ à $400 \mathrm{~g}(\mathrm{I} 0,56 \mu \mathrm{M}$ ADN/g SD à $4,99 \mu \mathrm{M} /$ g SD). Au-delà de $400 \mathrm{~g}$ (lots 6, 7 et 8), la concentration en ADN demeure stable.

La courbe représentative de l'évolution de la teneur en ARN/g SD en fonction du poids frais du muscle met également en évidence une diminution de $70 \mathrm{p}$. Ioo qui se manifeste jusqu'à ce que les truites aient atteint le poids de $850 \mathrm{~g}$; cette diminution de la concentration en ARN semble se faire par paliers successifs avec une alternance de chutes prononcées et de remontées de moindre importance, mais ces fluctuations ne sont pas statistiquement significatives.

Évolution en fonction du poids de certaines caractéristiques

\begin{tabular}{|c|c|c|c|c|c|c|}
\hline Numéro du lot & & 1 & & 2 & & 3 \\
\hline Poids vif moyen $(g)\left({ }^{1}\right)$ & 20,8 & $\pm 1,4$ & 50,8 & 2,9 & 114,1 & 7,6 \\
\hline Poids frais du muscle $(g), \ldots \ldots \ldots \ldots \ldots \ldots$ & 5,6 & $\pm 0,6$ & $1 / 4,0$ & 1,7 & 29,4 & 2,3 \\
\hline Poids sec et dégraissé du muscle $(g) \ldots \ldots \ldots \ldots$ & 0,88 & $\pm 0,08$ & 2,03 & 0,4 & 4,90 & 0,5 \\
\hline ARN /g sec et dégraissé $(\mu \mathrm{M}) \ldots \ldots \ldots \ldots$ & 29,7 & $\pm 3,3$ & 23,4 & 5,3 & 22,3 & 3,5 \\
\hline ARN total du muscle $(\mu \mathrm{M})$ & 26,3 & $\pm \quad 4,9$ & 47 & 7,2 & 109,6 & $\pm \quad 22,4$ \\
\hline ADN /g sec et dégraissé $(\mu \mathrm{M}) \ldots \ldots \ldots \ldots \ldots$ & 10,6 & $\pm 0,3$ & 9,9 & $\pm 2,05$ & $7, \mathbf{k}$ & 1,01 \\
\hline ADN total du muscle $(\mu \mathrm{M}) \ldots \ldots \ldots \ldots \ldots$ & 9,3 & $\pm 1,1$ & 20,0 & $\pm \quad 2,8$ & 36,2 & 5,0 \\
\hline ARN $/ A D N \ldots \ldots \ldots$ & 2,81 & $\pm \quad 0,29$ & 2,36 & 0,30 & 3,02 & 0,40 \\
\hline Poids frais $(\mathrm{mg})$ & $\mathbf{5 9 8}$ & $\pm 18,6$ & 700 & $\pm 101,5$ & 813 & $\pm 100,1$ \\
\hline Poids sec et dégraissé (mg) & 95 & $\pm 0,30$ & 100 & $\pm 21,0$ & $1 / 40$ & $\pm 19,20$ \\
\hline$A \overline{D N}$ total $(\mu \mathrm{M})$ & & & & & & \\
\hline
\end{tabular}

(1) Les valeurs moyennes sont accompagnées de leur écart-type. 


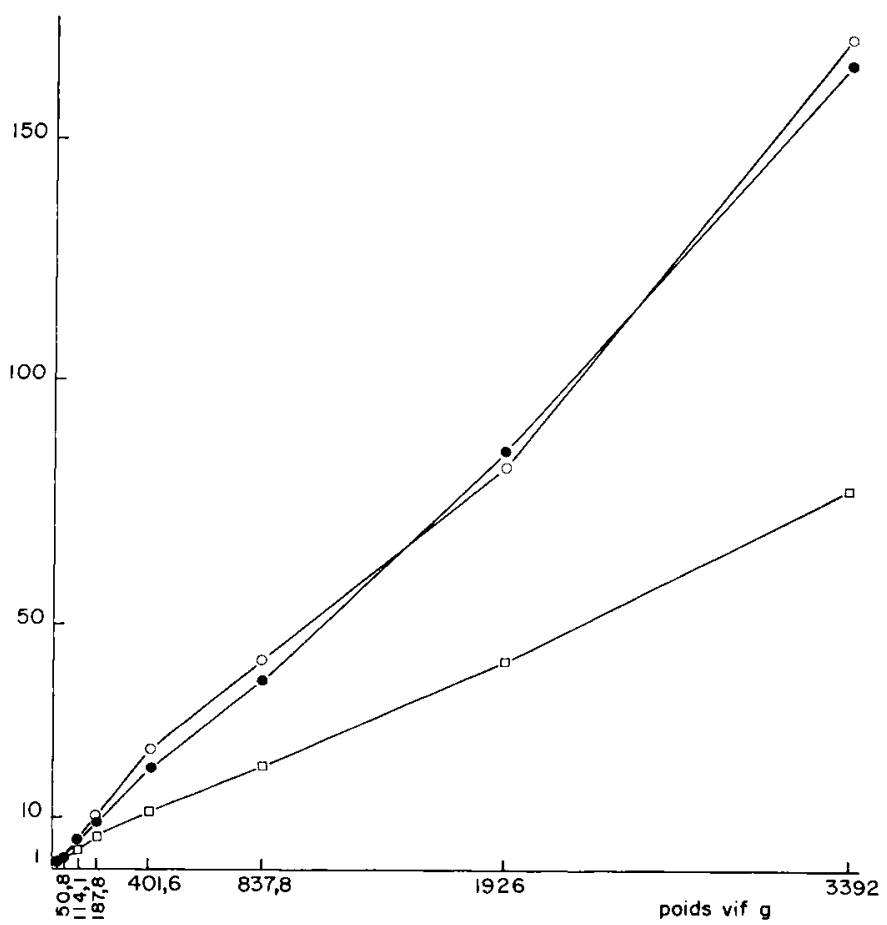

FIs. I. - Évolution du poids frais, du poids sec et dégraissé et de l'ADN total de la musculature épaxiale chez la truite Arc-en-ciel, en fonction du poids vif

En ordonnée, la valeur I est attribuée aux données correspondant aux truites du premier lot o Poids sec et dégraissé - Poids frais a ADN Total

AU 2

imiques de la musculature épaxiale de la truite Arc-en-Ciel

\begin{tabular}{|c|c|c|c|c|c|c|c|c|c|}
\hline & 4 & & 5 & & 6 & & 7 & & 8 \\
\hline 187,8 & $\pm 3,5$ & 401,6 & $\pm 17,1$ & 837,8 & $\pm 199,0$ & $1926 \mathrm{j}$ & \pm 100 & 3392 & $\pm 71^{\prime}, 1$ \\
\hline 54,2 & $\pm 3,6$ & 115,2 & $\pm 11,9$ & 216 & $\pm \quad 54,6$ & 476,8 & $\begin{array}{l} \pm \\
\pm\end{array}$ & 921 & $\pm 211,5$ \\
\hline 9,69 & $\pm 0,71$ & 21,9 & $\pm \quad 2,1$ & 37,7 & $\pm 10,7$ & 72,6 & $\begin{array}{l} \pm \quad 12,3 \\
\pm\end{array}$ & 150,3 & $\pm \quad 51,1$ \\
\hline 13,3 & $\pm 2,3$ & 16,2 & $\pm 1,9$ & 8,9 & $\pm \quad 2,25$ & 6,4 & $\pm \quad 0,63$ & 5,9 & $\pm \quad 0,4^{\prime} t$ \\
\hline 130,2 & $\pm 28,9$ & 357 & $\pm 66,{ }^{\prime}$ & 335,5 & $\pm 171,5$ & $46:, 5$ & $\pm 86,7$ & 888,3 & $\pm 239,9$ \\
\hline 6,6 & $\pm 0,5^{\prime}$ & 4,99 & $\begin{array}{ll} \pm & 0,10\end{array}$ & 5,2 & $\pm \quad 0,46$ & 5,5 & $\pm \quad 0,62$ & 4,8 & $\pm \quad 1,06$ \\
\hline 63,6 & $\pm 7,4$ & 109 & $\pm 13,7$ & 195,3 & $\pm 50,2$ & 398,6 & \pm 99 & $7 \% 4,5$ & $\pm 112,19$ \\
\hline 2,02 & $\pm \quad 0,31$ & 3,25 & $\pm \quad 0,3 x$ & $1,7:$ & $\pm \quad 0,61$ & 1,16 & 土 $\quad 0,16$ & 1,22 & $\pm \quad 0,23$ \\
\hline 850 & $\pm 68,9$ & 1057 & $\pm 73,6$ & 1106 & $\pm 88,7$ & 1196 & $\pm 166,5$ & 1271 & \pm 200 \\
\hline 150 & $\pm 12,9$ & 200 & $\pm 18,7$ & 193 & $\pm \quad 17,9$ & 182 & 土 19,9 & 207 & $\pm \quad 40,32$ \\
\hline
\end{tabular}


L'évolution du rapport ARN/ADN (fig. 2 A) met en évidence deux phases successives ; lorsque le poids des truites s'accroît de $20 \mathrm{~g}$ à $400 \mathrm{~g}$, le rapport ARN / ADN augmente jusqu'à la valeur 3,25. Lorsque le poids des truites dépasse $400 \mathrm{~g}$, ce rapport diminue rapidement pour se stabiliser autour de $\mathrm{I}, 20$. Au cours de la phase ascendante les valeurs du rapport ARN/ADN présentent des oscillations qui rappellent celles déjà observées dans l'évolution de la concentration en ARN.

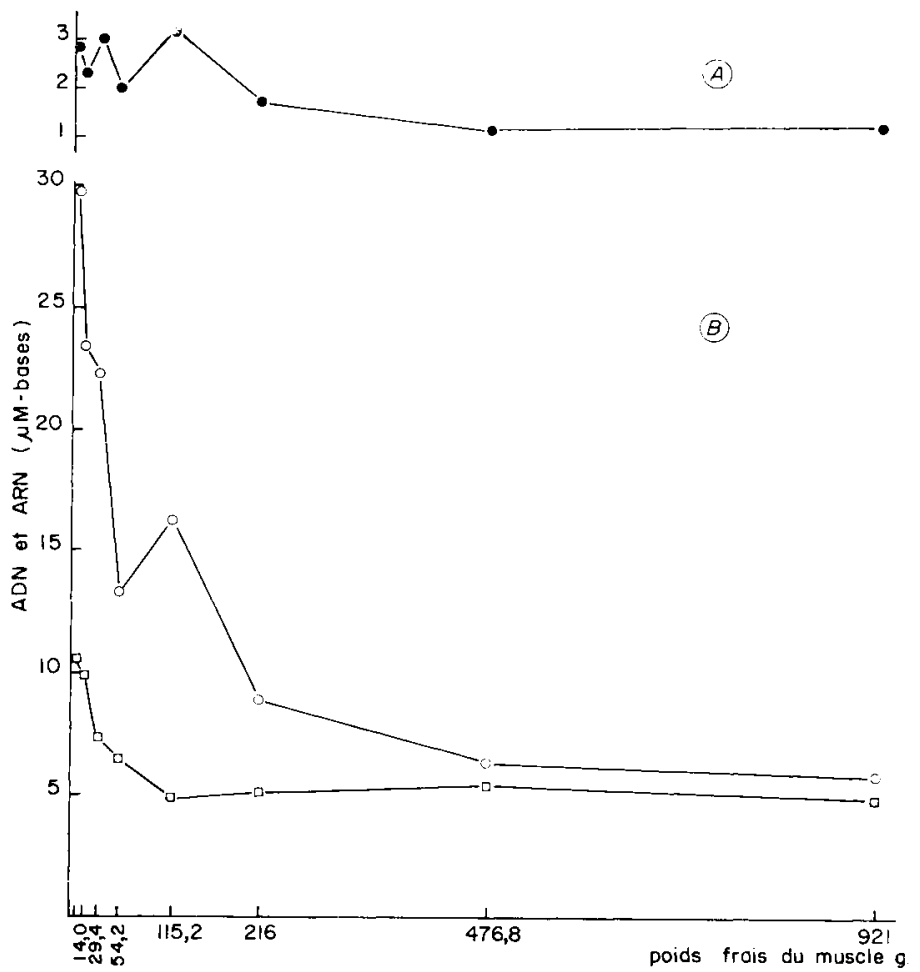

Fig. 2. - Évolution en fonction du poids frais du muscle: A du rapport $\frac{A R N}{A D N}$,

$\mathrm{B}$ de la quantité d'ARN et $A D N$ contenu dans 1 gramme sec et dégraissé

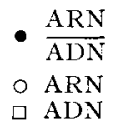

b) Évolution de l'ARN et de l'ADN total (fig. 3)

Sur l'ensemble de l'intervalle considéré, la quantité d'ADN total atgmente deux fois moins vite que le poids du muscle. Ainsi, lorsque ce dernier est multiplié par I65, la quantité d'ADN est multiplié par 78 . La courbe représentative de cette évolution est pratiquement linéaire.

L'ARN total est multiplié par I4 lorsque le poids des truites passe de $20 \mathrm{~g}$ à $400 \mathrm{~g}$. Cette augmentation semble marquer un arrêt jusqu'à $840 \mathrm{~g}$ pour reprendre à un degré moindre chez les poissons d'un poids supérieur. 


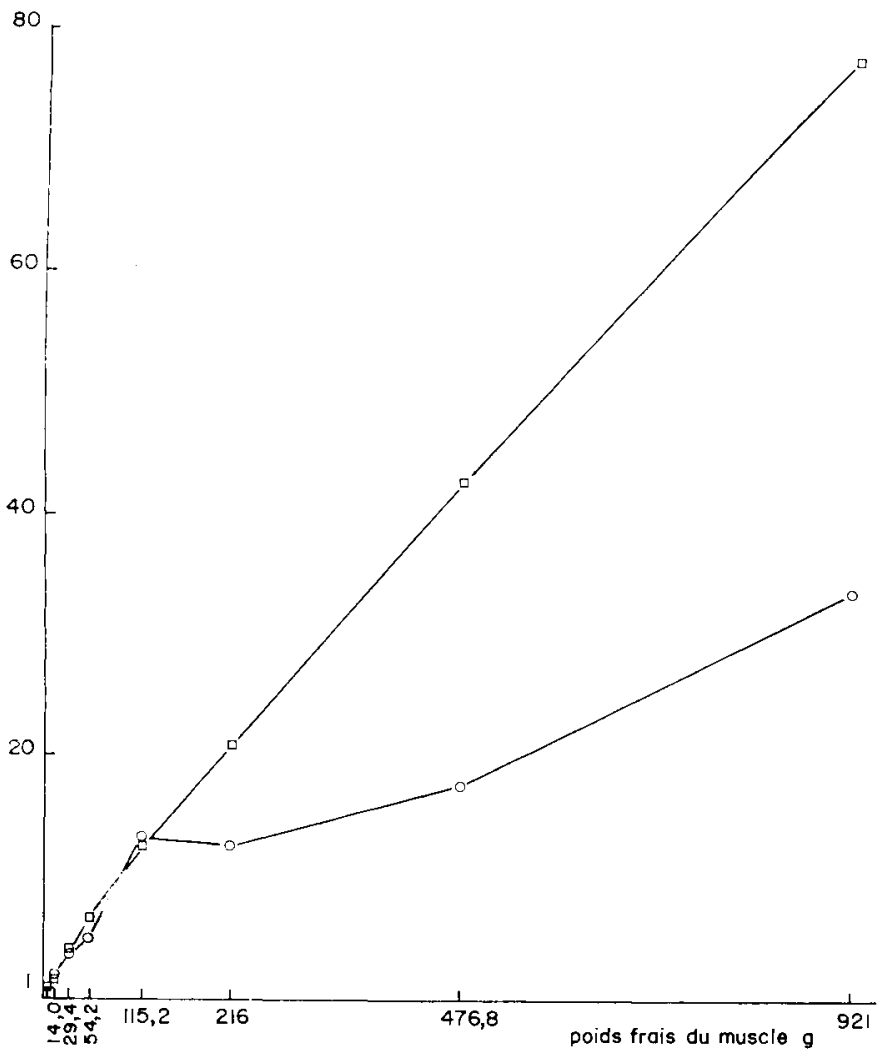

FIG. 3. - Evolution, en fonction du poids frais, de l'ADN total et de i'ARN total du muscle de la truite Arc-en-ciel

En ordonnée, la valeur I est attribuée aux données correspondant aux truites de $20 \mathrm{~g}$ $\square$ ADN total

- ARN total

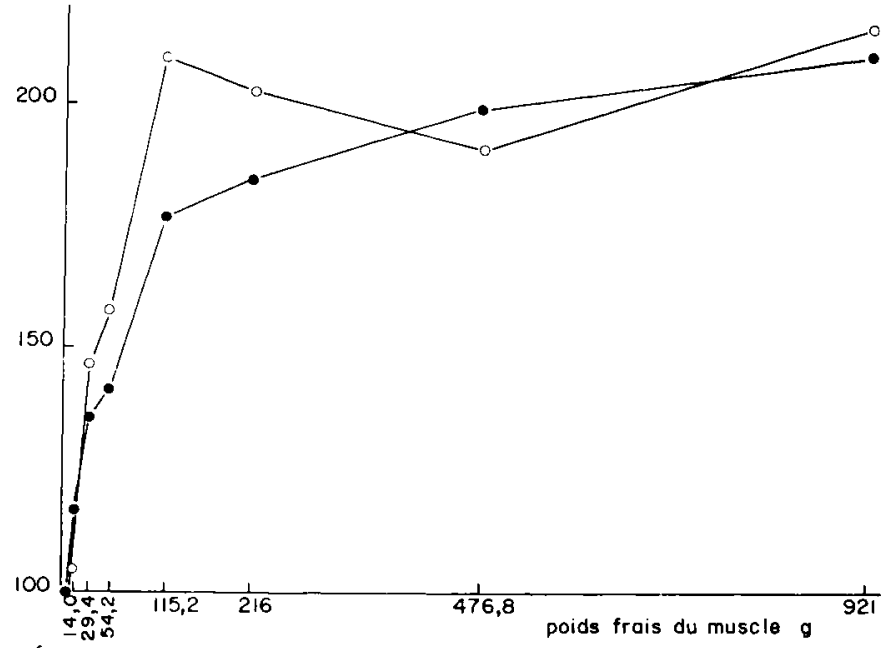

FIG. 4. - Évolution de la taille des cellules (poids frais/ADN total) et de la quantité de protéines par cellule (poids sec et dégraissé/ADN total) en fonction du poids frais $d u$ muscle.

En ordonnée la valeur roo est attribuée aux données correspondant aux truites de $20 \mathrm{~g}$

- Poids sec et dégraissé/ADN total

- Poids frais/ADN total 
c) Évolution des rapports poids frais/ADN, poids sec et dégraissé/ADN (fig. 4).

La valeur du rapport poids frais/ADN est multipliée par $I, 8$ lorsque le poids des truites passe de $20 \mathrm{~g}$ à $400 \mathrm{~g}$. Au-delà, l'augmentation de ce rapport est plus faible quoique sensible. Sur l'ensemble de l'intervalle cette valeur est multipliée par 2,I2.

Le rapport PSD/ADN augmente plus rapidement que le poids frais/ADN jusqu'à ce que les truites atteignent $400 \mathrm{~g}$. La valeur de ce rapport a en effet doublé $(\times 2,10)$ lorsque les poissons sont arrivés à ce poids. Au-delà, la valeur du rapport semble se stabiliser à ce niveau.

\section{DISCUSSION}

\section{I. - Remarques générales}

\section{Caractéristiques des animaux}

Les truites des cinq premiers lots ont été sacrifiées au cours de la période estivale tandis que celles des lots 6,7 et 8 l'ont été durant 1'hiver. A l'intérieur de ces deux groupes les caractéristiques sont donc comparables entre elles. Comme les résultats ne marquent pas de discontinuité lorsque l'on passe du lot 5 au lot 6 , il semble possible d'établir des comparaisons sur l'ensemble de l'intervalle.

\section{Infuence de l'ADN sanguin}

Les érythrocytes de poissons étant nucléés, la quantité d'ADN sanguin pourrait perturber les résultats concernant la concentration de l'ADN musculaire. Le muscle de truite contient :

- I,7 $\mathrm{ml}$ de sang par Ioo $\mathrm{g}$ de poids frais (STEVENS, I968) soit :

$\mathrm{I}, 7 \times \mathrm{I}, 5 \mathrm{IO}^{6}=2,55 \mathrm{IO}^{6}$ érythrocytes/ $\mathrm{IO0} \mathrm{g}$ de poids frais (SMITH, I 968 ) soit :

$2,55 \mathrm{IO}^{8} \times 4,9 \mathrm{IO}^{8}=\mathrm{I} 2,49 \mu \mathrm{g} \mathrm{ADN} / \mathrm{IOO} g$ de poids frais

(VENDRELY et VENDRELY, I963)

or, le muscle de truite renferme :

$57,4 \mathrm{mg} \mathrm{ADN} / \mathrm{I}$ oo $\mathrm{g}$ de poids frais pour le poids de $20 \mathrm{~g}$ 26,6 $\mathrm{mg}$ ADN/roo g de poids frais pour le poids de $3400 \mathrm{~g}$

donc le rapport $\mathrm{ADN}$ sanguin/ADN total du muscle varie entre $\mathrm{I} / 4$ o0o et $\mathrm{I} / 2000$ ce qui est négligeable.

\section{II. - Multiplication et grandissement cellulaires (tab1. 3)}

Un calcul simple, élaboré par Durand et al. (1965), permet de mesurer l'intervention relative de la multiplication cellulaire (hyperplasie) et du grandissement cellulaire (hypertrophie) au cours des différentes étapes de la croissance. Appliqué à la croissance du muscle de la Truite, ce calcul montre que l'hyperplasie contribue 


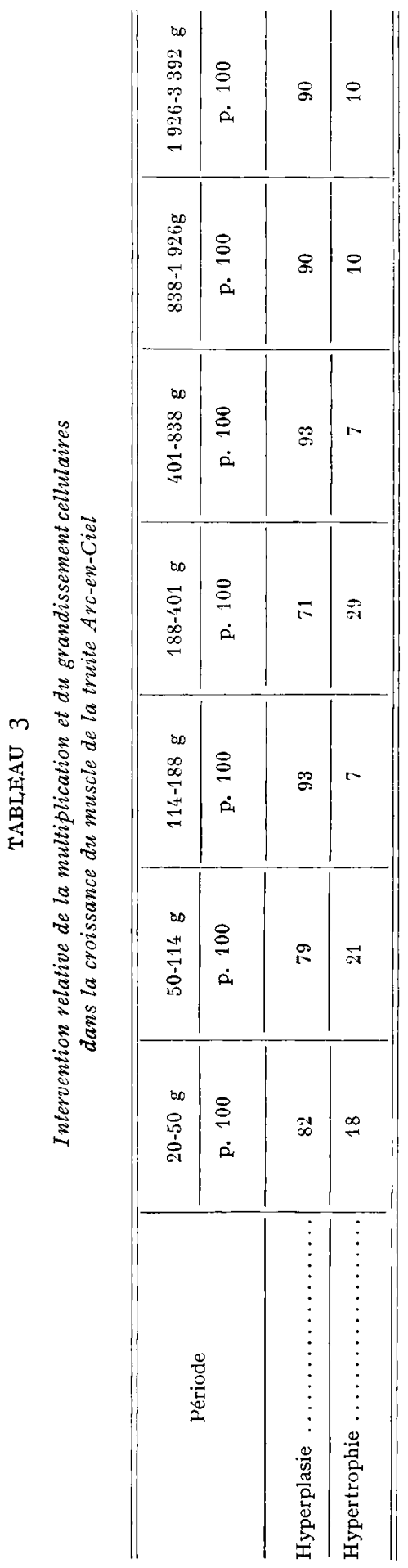


toujours pour plus de $70 \mathrm{p}$. Ioo à la croissance pondérale. Son intervention a tendance à augmenter lorsque les truites croissent pour se stabiliser autour de go p. roo dès qu'elles atteignent le poids de $400 \mathrm{~g}$.

Chez les Mammifères, chez lesquels cela a été étudié (rat-porc), le rôle de l'hyperplasie n'excède jamais $80 \mathrm{p}$. Ioo et a tendance à diminuer au cours du développement musculaire de telle sorte que, lorsque les animaux atteignent la taille qui correspond à la fin de la phase de croissance autoaccélérée, le rôle principal est dévolu à l'hypertrophie (DURAND et al., I965, I967). Dans le présent travail, nous n'avons rien constaté de tel chez ce poisson qu'est la truite, sauf entre les poids de rgo g et $400 \mathrm{~g}$. Les cellules grandissent rapidement jusqu'au poids de $400 \mathrm{~g}$. A ce stade, leur taille représente $75 \mathrm{p}$. roo de la taille maximale observée. Le ralentissement de l'hypertrophie noté par la suite, peut être attribué à deux causes : d'une part, on peut penser que les cellules du muscle perdent en partie la possibilité de s'accroître en taille, d'autre part, étant donné que ce ralentissement de 1'hypertrophie se remarque dès que le poids des truites excède $400 \mathrm{~g}$, peut intervenir une fonte musculaire consécutive à l'élaboration des produits sexuels. Ce phénomène observé chez le Saumon par GREENE (I9I9) et IDLER et TsUYUKI (I958) ne semble pas cependant intervenir d'une façon importante chez la Truite Arc-en-Ciel ; en effet, la légère augmentation de $1^{\prime} \mathrm{ADN} /$ poids sec dégraissé observée chez les truites correspondantes n'est pas statistiquement significative.

Une comparaison interspécifique nous montre que les quantités de protéines et de matière fraîche que contrôle l'unité d'ADN du muscle des truites de $20 \mathrm{~g}$ et $400 \mathrm{~g}$ correspondent à celles du muscle (biceps femoris) de porcs pesant $20 \mathrm{~kg}$ et I50 $\mathrm{kg}$ respectivement (DuRAND et al., Ig67).

\title{
III. - Activité cellulaire
}

Comme cela a déjà été observé sur le Rat (DEvi et al., I963, Durand et al., I965), nous avons constaté que la valeur du rapport ARN/ADN passe par un maximum pour diminuer chez les animaux âgés. Il faut cependant noter que, dans les conditions expérimentales, cette évolution peut être accélérée par le fait que les truites des 3 derniers lots ont été sacrifiées au cours de la période normale de reproduction; en effet, CREELMAN et TOMIrNSON (I959) ont déjà remarqué que la valeur de ce rapport diminue lors de l'élaboration des produits sexuels. Aussi l'interprétation de ce phénomène ainsi que celle đu ralentissement du grandissement cellulaire observé au-delà du poids de $400 \mathrm{~g}$ sont rendues délicates du fait de l'interférence possible des modifications induites par la maturation sexuelle. Un travail est actuellement en cours pour éclaircir ces points.

Reçu pour publication en mars 1970.

\section{SUMMARY}

\author{
CHANGES IN NUCL,EIC ACID CONTENT OF EPAXIAL MUSCUL,ATURE \\ DURING GROWTH OF RAINBOW TROUT SALMO GAIDNERII; \\ RESPECTIVE ROLES OF INCREASE IN NUMBER AND SIZE OF CELLS
}

Growth of the lateral dorsal muscles of the rainbow trout were studied by the following biochemical methods : 
- measurement of the deoxyribonucleic acid (DNA) content, the amount of which is proportional to the number of cells;

- measurement of variations in the ratio of fresh weight to DNA, proportional to the size of the cells;

- measurement of variations in the ratio of ribonucleic acid (RNA) to DNA, which shows cellular activity.

The purine bases of DNA and the nucleotides of RNA were estimated by colum chromatography after extraction by the method of SCHMIDT and THANNHAUSER.

When the weight of the trout was multiplied by 163 (growth from $20 \mathrm{~g}$ to $3400 \mathrm{~g}$ ) it was shown that;

- weight of the muscle was multiplied by 165 ;

- the number of cells was multiplied by 78 ; the increase in cell number (hyperplasia) continued throughout the interval studied;

- the cells doubled in size; they had reached 75 per cent of final size when the trout weighed $400 \mathrm{~g}$, after which weight the increase in cell size (hypertrophy) slowed down.

- hyperplasia always accounted for $7^{\circ}$ per cent of the increase in weight of the muscle. Its relative importance was greater than go per cent when the weight of trout passed $400 \mathrm{~g}$.

These results are compared with those obtained with rats and pigs. They show that the mechanisms of growth fish differ considerably from those which regulate the growth of mammals.

\section{RÉFÉRENCES BIBLIOGRAPHIQUES}

Berdyshev G. D., Korotaev G. K., Bojarskikh G. V., Vanushin B. F., ig67. Composition en nucléotides du DNA et duRNA des tissus somatiques de l'Oncorhynchus gorbuscha et sa variation pendant le frai. Biokhimia 32, 988-993.

Bertalanffy L., Von, I960. Principles and theory of growth in : Fundamental aspects of normal and malignant growth Nowinski W. W. Ed. (Elsevier, New York).

Boivis A., Vendrely R. Vendrely C., I948. L'acide désoxyribonucléique du noyau cellulaire dépositaire des caractères héréditaires ; arguments d'ordre analytique. C. R. Acad. Sci., 226, ro6r-Io62.

Chargaff E., Lipshitz R., I953. Composition of mammalian desoxyribonucleic acids. J. Amer. Chem. Soc., 75, 3658-366r.

Creelmax V. M., Tomlinson N., I959. Biochemical studies on Sockeye salmon during spawning migration. VI. Ribonucleic acid and deoxyribonucleic acid. J. Fish. Res. Bd. Can., 16, 42I-428.

Devi A., Munundan M. A., SRivastava U., Sarkar N. K., Ig63. The effect of age on the variations of deoxyribonucleic acid and total nucleotides in liver, brain and muscle of rat. Expl. cell Res., $32,242-250$.

Durand G., Falconneau G., Penot E., 1965. Étude biochimique de la croissance de l'intestin grêle, du foie et de la carcasse du Rat; rôles respectifs de la multiplication et du grandissement cellulaires. Ann. Biol. anim. Bioch. Biophys., 5, 163-187.

Durand G., Fatconveau G., Penot E., i967. Évolution de la teneut en acides nucléiques et en protéines du muscle chez le Porc au cours de la croissance postnatale. C. R, Acad. Sci. Paris, 264, 16401643.

Durand G., Fauconneau G., Penot E., I969. Répartition des cellules entre les tissus du Rat adulte, préalablement soumis à une sous-nutrition énergétique temporaire à deux stades de la croissance. Ann. Biol. anim. Bioch. Biophys., 9, 55-73.

ENESco M., I959. Increase in number of nuclei and the mean cell size during postnatal growth of the heart of male Sherman rats. Ant. Rec., 133, 272.

Greene C. W., rgrg. Biochemical changes in the muscle tissue of King salmon during the fast of spawning migration. J. Biol. chem., 39, 435-456.

IDLER D. R., TsuYuki H., I958. Biochemical studies on Sockeye salmon during spawning migration. I. Physical measurements, plasma cholesterol, and electrolyte levels. Can. J. Biochem. Physiol., 36, $783-79 \mathrm{I}$.

LuQuet P., I970. Étude des divers composés azotés des salmonidés : protéines, azote protéique, acides nucléiques. Facteurs de variations. Recherches d'hydrobiologie continentale (sous presse).

Moss F. P., Srmmonds R. A., McNary H. W., I964. The growth and composition of skeletal muscle in the chicken. 2. Relationship between muscle weight and the number of nuciei. Poultry Sci., 43, IO86-IOgI.

Paloheimo J. E., Dickie L. M., I965. Food and growth of fishes. I. A growth curve derived from experimental data. J. Fish Res. Bd. Can., 22, 52 I-542.

Schmidt G., Thannhauser S. J., I945. A method for the determination of the desoxyribonucleic acid, ribonucleic acid, and phosphoproteins in animal studies. J. Biol. chem., 161, 83-89. 
Sмiтн C. F., 1968. Hematological changes in coho salmon fed a folic acid deficient diet. J. Fish. Res. Bd. Can., 25., $15 \mathrm{I}-\mathrm{I} 56$.

Stevens Don E., I 968 . The effect of exercice on the distribution of blood to various organs in rainbow trout. Comp. Biochem. Physiol., 25, 6r5-626.

Strunz K., Lenkeit W., I963. Die Stickstoff und Nukleinsäure-Verteilung im Ferkel-körper unter dem Einfluss der Proteinzufuhr (Vorläufige Ergebnisse). 2. - Mitteilung;: Der DNS und RNS-Gehalt in Skeletmuskulatur, Herzmuskulatur, Niere, Leber und Milz. Z. Tierphys. Tievernähr, Futterm., 18, 285-296.

VeNDRELY R., VendRELY C., I953. Arginine and deoxyribonucleic acid content of erythrocyte nuclei and sperms of some species of fishes. Nature, 172, 30-3r. 